\title{
JOINT SPECTRA AND JOINT NUMERICAL RANGES FOR PAIRWISE COMMUTING OPERATORS IN BANACH SPACES
}

\author{
by VOLKER WROBEL
}

(Received 27 June, 1986)

In a recent paper $M$. Cho [5] asked whether Taylor's joint spectrum $\sigma\left(a_{1}, \ldots, a_{n} ; X\right)$ of a commuting $n$-tuple $\left(a_{1}, \ldots, a_{n}\right)$ of continuous linear operators in a Banach space $X$ is contained in the closure $V\left(a_{1}, \ldots, a_{n} ; X\right)^{-}$of the joint spatial numerical range of $\left(a_{1}, \ldots, a_{n}\right)$. Among other things we prove that even the convex hull of the classical joint spectrum $S p\left(a_{1}, \ldots, a_{n} ;\left\langle a_{1}, \ldots, a_{n}\right\rangle\right)$, considered in the Banach algebra $\left\langle a_{1}, \ldots, a_{n}\right\rangle$, generated by $a_{1}, \ldots, a_{n}$, is contained in $V\left(a_{1}, \ldots, a_{n} ; X\right)^{-}$.

0. Notation. Throughout this paper $X$ will always denote a Banach space over the complex numbers $\mathbb{C}$, and $L(X)$ will denote the Banach algebra of all continuous linear operators on $X$. Operator will always mean continuous linear operator. $X^{\prime}$ denotes the dual space of $X$ and for $a \in L(X)$ we let $a^{\prime}$ denote the dual operator. Given a subset $B \subset X$ we let $B^{-}$denote the closure of $B$.

1. Joint spectra. Let $\left(a_{1}, \ldots, a_{n}\right) \in L(X)^{n}$ denote an $n$-tuple of pairwise commuting operators, and let $A$ denote a closed unital subalgebra containing $a_{1}, \ldots, a_{n}$. In accordance with Bonsall and Duncan $\left[1\right.$, p. 24] the joint spectrum $\operatorname{Sp}\left(a_{1}, \ldots, a_{n} ; A\right)$ of $a_{1}, \ldots, a_{n}$ with respect to $A$ consists of those points $z=\left(z_{1}, \ldots, z_{n}\right) \in \mathbb{C}^{n}$ such that either

$$
\sum_{i=1}^{n}\left(z_{i}-a_{i}\right) A
$$

is a proper right ideal in $A$ or

$$
\sum_{i=1}^{n} A\left(z_{i}-a_{i}\right)
$$

is a proper left ideal in $A$. Suitable choices for $A$ are $L(X)$ [8], the commutant algebra $\left\{a_{1}, \ldots, a_{n}\right\}^{c}$ of $a_{1}, \ldots, a_{n}$ in $L(X)[\mathbf{1 0}]$, the bicommutant algebra $\left\{a_{1}, \ldots, a_{n}\right\}^{c c}$ [7] or the Banach algebra $\left\langle a_{1}, \ldots, a_{n}\right\rangle$ generated by $a_{1}, \ldots, a_{n}$ (Gelfand).

J. L. Taylor [10] considers a spatial version of joint spectrum denoted by $\sigma\left(a_{1}, \ldots, a_{n} ; X\right)$ throughout.

Moreover we consider the following concepts. By definition a point $z=$ $\left(z_{1}, \ldots, z_{n}\right) \in \mathbb{C}^{n}$ belongs to the joint point spectrum $\operatorname{Po}\left(a_{1}, \ldots, a_{n} ; X\right)$ or the joint approximate point spectrum $A P \sigma\left(a_{1}, \ldots, a_{n} ; X\right)$, if there exists a vector $x \neq 0$ or a sequence $\left(x_{n}\right)_{n \in \mathbb{N}}$ with $\left\|x_{n}\right\|=1$ such that $a_{i} x=z_{i} x$ for $1 \leq i \leq n$ and

$$
\begin{gathered}
\left\|a_{i} x_{n}-z_{i} x_{k}\right\| \rightarrow 0 \text { as } k \rightarrow \infty \text { for } 1 \leq i \leq n, \\
\text { Glasgow Math. J. } 30 \text { (1988) 145-153. }
\end{gathered}
$$


respectively. Finally, $C \sigma\left(a_{1}, \ldots, a_{n} ; X\right):=P \sigma\left(a_{1}^{\prime}, \ldots, a_{n}^{\prime} ; X^{\prime}\right)$ and $A C \sigma\left(a_{1}, \ldots, a_{n} ; X\right):=$ $A P \sigma\left(a_{1}^{\prime}, \ldots, a_{n}^{\prime} ; X^{\prime}\right)$ denote the joint compression spectrum and the joint approximate compression spectrum, respectively.

Obviously, we have

$$
A P \sigma\left(a_{1}, \ldots, a_{n} ; X\right) \cup A C \sigma\left(a_{1}, \ldots, a_{n} ; X\right) \subseteq S p\left(a_{1}, \ldots, a_{n} ; L(X)\right) \cap \sigma\left(a_{1}, \ldots, a_{n} ; X\right) .
$$

Moreover, by [10]

$$
\sigma\left(a_{1}, \ldots, a_{n} ; X\right) \subseteq S p\left(a_{1}, \ldots, a_{n} ;\left\{a_{1}, \ldots, a_{n}\right\}^{c}\right)
$$

with proper inclusion in general.

We start with a summary of polynomial spectral mapping theorems.

1.1. ThEOREM. Let $\left(a_{1}, \ldots, a_{n}\right) \in L(X)^{n}$ denote an $n$-tuple of pairwise commuting operators, and let $Q$ denote a polynomial in $n$ variables. Then the following spectral mapping theorems hold:

(i) $Q\left(\operatorname{Sp}\left(a_{1}, \ldots, a_{n} ; A\right)\right)=\operatorname{Sp}\left(Q\left(a_{1}, \ldots, a_{n}\right) ; A\right)$,

where A denotes a unital Banach subalgebra of $L(X)$ containing $a_{1}, \ldots, a_{n}$;

(ii) $Q\left(\sigma\left(a_{1}, \ldots, a_{n} ; X\right)\right)=\sigma\left(Q\left(a_{1}, \ldots, a_{n}\right) ; X\right)$

$$
=\operatorname{Sp}\left(Q\left(a_{1}, \ldots, a_{n}\right) ; L(X)\right)
$$

(iii) $Q\left(A P \sigma\left(a_{1}, \ldots, a_{n} ; X\right)\right)=A P \sigma\left(Q\left(a_{1}, \ldots, a_{n}\right) ; X\right)$.

For (i) see [8], for (ii) see [11] and for (iii) see [6].

Given a compact subset $K \subset \mathbb{C}^{n}$ let

$$
\text { p.c.h. }(K):=\left\{z \in \mathbb{C}^{n}:|Q(z)| \leq \max _{t \in K}|Q(t)| \text { for all polynomials } Q\right\}
$$

denote the polynomially convex hull of $K$. If $K=$ p.c.h. $(K)$, then $K$ is said to be polynomially convex. It is a well-known fact in classical Banach algebra theory, that $S p\left(a_{1}, \ldots, a_{n} ;\left\langle a_{1}, \ldots, a_{n}\right\rangle\right)$ is a polynomially convex compact set. (See $[3$, p. 101] or $[12$, p. 44]).

Observe that if $n=1$ then polynomially convex means having a connected complement. But if $n>1$ there is no topological description of polynomially convex sets. Indeed let us note the following fact which gives a general context of Wermer's remark and example in [12, p. 36].

1.2. REMARK. Each compact subset of $\mathbb{C}^{n}$ is homeomorphic to a polynomially convex set in $\mathbb{C}^{2 n}$. More precisely: given a compact subset $K$ of $\mathbb{C}^{n}$, the set

$$
\tilde{K}:=\left\{(z, \bar{z}) \in \mathbb{C}^{2 n}: z \in K\right\}
$$

is polynomially convex, where "bar" denotes complex conjugation. 
Proof. $1^{\circ}$ Observe that given an $n$-tuple $\left(a_{1}, \ldots, a_{n}\right) \in L(H)^{n}$ of pairwise commuting normal operators on a Hilbert space $H$, we have from [7]

$$
\begin{aligned}
S p\left(a_{1}, \ldots, a_{n} ;\left\{a_{1}, \ldots, a_{n}\right\}^{c c}\right)=A P \sigma\left(a_{1}, \ldots, a_{n} ; H\right) & \\
& =S p\left(a_{1}, \ldots, a_{n} ;\left\langle a_{1}, \ldots, a_{n}, a_{1}^{*}, \ldots, a_{n}^{*}\right\rangle\right),
\end{aligned}
$$

$a^{*}$ denoting the Hilbert space adjoint of $a$. To see the last non-trivial inclusion, let $z=\left(z_{1}, \ldots, z_{n}\right) \notin A P \sigma\left(a_{1}, \ldots, a_{n} ; H\right)$. Then the positive operator $\sum_{i=1}^{n}\left(a_{i}-z_{i}\right)^{*}\left(a_{i}-z_{i}\right)$ is a topological monomorphism and therefore a bijection. This proves that

$$
z \notin S p\left(a_{1}, \ldots, a_{n} ;\left\langle a_{1}, \ldots, a_{n}, a_{1}^{*}, \ldots, a_{n}^{*}\right\rangle\right) .
$$

$2^{\circ}$ Next recall that $\operatorname{Sp}\left(a_{1}, \ldots, a_{n} ;\left\langle a_{1}, \ldots, a_{n}, a_{1}^{*}, \ldots, a_{n}^{*}\right\rangle\right)$ is the projection of $S p\left(a_{1}, \ldots, a_{n}, a_{1}^{*}, \ldots, a_{n}^{*} ;\left\langle a_{1}, \ldots, a_{n}, a_{1}^{*}, \ldots, a_{n}^{*}\right\rangle\right)$ onto the first $n$ coordinates (compare Zelasko [15]).

$3^{\circ}$ Note that for an $n$-tuple $\left(a_{1}, \ldots, a_{n}\right)$ of diagonal operators

we have

$$
a_{k}:=\operatorname{diag}\left(\left(\alpha_{j k}\right)_{j \in \mathbb{N}}\right)(1 \leq k \leq n) \text { on } l^{2}
$$

$$
A P \sigma\left(a_{1}, \ldots, a_{n} ; l^{2}\right)=\left\{\alpha^{(i)}: i \in \mathbb{N}\right\}^{-},
$$

where $\alpha^{(i)}=\left(\alpha_{i 1}, \alpha_{i 2}, \ldots, \alpha_{i n}\right)(i \in \mathbb{N})$. Especially given a compact subset $K$ of $\mathbb{C}^{n}$ let $\left\{\alpha^{(i)}: i \in \mathbb{N}\right\}$ denote a dense subset of $K$ and define diagonal operators as above. Then $K=A P \sigma\left(a_{1}, \ldots, a_{n} ; l^{2}\right)[4,5.1]$.

$4^{\circ}$ Finally putting together $1^{\circ}-3^{\circ}$ we get the desired result.

The following lemma, which also has been used in [13] especially states as its main consequence that the polynomially convex hulls of almost all joint spectra coincide with $S p\left(a_{1}, \ldots, a_{n} ;\left\langle a_{1}, \ldots, a_{n}\right\rangle\right)$.

1.3. Lemma. Let $\left(a_{1}, \ldots, a_{n}\right) \in L(X)^{n}$ denote an $n$-tuple of pairwise commuting operators. Let $K \subseteq S p\left(a_{1}, \ldots, a_{n} ;\left\langle a_{1}, \ldots, a_{n}\right\rangle\right)$ denote a nonempty compact set such that for every polynomial $Q$ in $n$ variables we have

Then

$$
\begin{aligned}
\max _{t \in K}|Q(t)| & =r\left(Q\left(a_{1}, \ldots, a_{n}\right) ; L(X)\right) \\
: & =\max \left\{|z|: z \in \operatorname{Sp}\left(Q\left(a_{1}, \ldots, a_{n}\right) ; L(X)\right)\right\} .
\end{aligned}
$$

$$
\text { p.c.h. }(K)=S p\left(a_{1}, \ldots, a_{n} ;\left\langle a_{1}, \ldots, a_{n}\right\rangle\right) \text {. }
$$

Proof. Obviously we have

$$
\text { p.c.h. }(K) \subseteq S p\left(a_{1}, \ldots, a_{n} ;\left\langle a_{1}, \ldots, a_{n}\right\rangle\right) \text {. }
$$

On the other hand let $z=\left(z_{1}, \ldots, z_{n}\right) \notin$ p.c.h. $(K)$. By definition there exists a polynomial $Q$ such that

$$
|Q(z)|>\max _{t \in K}|Q(t)|=r\left(Q\left(a_{1}, \ldots, a_{n}\right) ; L(X)\right) .
$$


Therefore

$$
b:=\sum_{j=0}^{\infty} Q\left(a_{1}, \ldots, a_{n}\right)^{j} Q(z)^{-j-1} \in\left\langle a_{1}, \ldots, a_{n}\right\rangle
$$

is the inverse of $Q(z)-Q\left(a_{1}, \ldots, a_{n}\right)$. On the other hand

$$
Q(z)-Q\left(a_{1}, \ldots, a_{n}\right)=\sum_{j=1}^{n}\left(z_{j}-a_{j}\right) Q_{j}\left(a_{1}, \ldots, a_{n}\right),
$$

with suitable polynomials $Q_{j}$. Multiplication with $b$ gives $z \notin S p\left(a_{1}, \ldots, a_{n}\right.$; $\left.\left\langle a_{1}, \ldots, a_{n}\right\rangle\right)$ and hence the desired result.

Given a compact subset $K \subset \mathbb{C}^{n}$ let $\operatorname{conv}(K)$ denote the convex hull, and let $\operatorname{ext}(K)$ denote the extreme points of $\operatorname{conv}(K)$. Since p.c.h. $(K) \subseteq \operatorname{conv}(K)$, we have p.c.h. $(\operatorname{conv}(K))=\operatorname{conv}($ p.c.h. $(K))$, and consequently the following result is an immediate consequence of 1.1 and 1.3 .

1.4. Corollary. Let $\left(a_{1}, \ldots, a_{n}\right) \in L(X)^{n}$ denote an $n$-tuple of pairwise commuting operators. Then we have

$$
\begin{aligned}
& \operatorname{ext}\left(\operatorname{Sp}\left(a_{1}, \ldots, a_{n} ;\left\langle a_{1}, \ldots, a_{n}\right\rangle\right)\right)=\operatorname{ext}\left(\sigma\left(a_{1}, \ldots, a_{n} ; X\right)\right) \\
= & \operatorname{ext}\left(\operatorname{Sp}\left(a_{1}, \ldots, a_{n} ; L(X)\right)\right)=\operatorname{ext}\left(A P \sigma\left(a_{1}, \ldots, a_{n} ; X\right)\right) \\
= & \operatorname{ext}\left(A C \sigma\left(a_{1}, \ldots, a_{n} ; X\right)\right) .
\end{aligned}
$$

Especially,

$$
\operatorname{ext}\left(\sigma\left(a_{1}, \ldots, a_{n} ; X\right)\right)=\operatorname{ext}\left(A P \sigma\left(a_{1}, \ldots, a_{n} ; X\right) \cap A C \sigma\left(a_{1}, \ldots, a_{n} ; X\right)\right) .
$$

REMARK. If $n=1$, then $\partial \sigma\left(a_{1} ; X\right) \subseteq A P \sigma\left(a_{1} ; X\right) \cap A C \sigma\left(a_{1} ; X\right)$ ( $\partial$ denoting the boundary of .), whereas for $n=2$ neither $\partial \sigma\left(a_{1}, a_{2} ; X\right) \subseteq A P \sigma\left(a_{1}, a_{2} ; X\right)$ nor $\partial \sigma\left(a_{1}, a_{2}, X\right) \subseteq A C \sigma\left(a_{1}, a_{2} ; X\right)$ is true in general $([13,2.5])$. Therefore $1.3\left({ }^{* *}\right)$ may be regarded as a substitute for $\partial \sigma \subseteq A P \sigma \cap A C \sigma$ in the case $n \geq 2$. ( $\left.{ }^{* *}\right)$ also generalizes a previous result $[13,2.8]$ and simplifies its proof considerably.

In section 2 we shall need a concept of joint spectral radius for an $n$-tuple $\left(a_{1}, \ldots, a_{n}\right)$ of pairwise commuting operators.

Given a compact set $K \in \mathbb{C}^{n}$ let

$$
\|K\|_{2}:=\max \left\{\left(\sum_{j=1}^{n}\left|z_{j}\right|^{2}\right)^{1 / 2}:\left(z_{1}, \ldots, z_{n}\right) \in K\right\} .
$$

Since a continuous convex function takes its maximum on a compact set $K$ in $\operatorname{ext}(K)$, we obtain a notion of joint spectral radius $r\left(a_{1}, \ldots, a_{n}\right)$ which is independent of the underlying concept of joint spectrum as far as the assumptions of 1.3 are fulfilled.

1.5. Definition. Let $\left(a_{1}, \ldots, a_{n}\right) \in L(X)^{n}$ denote an $n$-tuple of pairwise commuting operators. Then

$$
r\left(a_{1}, \ldots, a_{n}\right):=\|K\|_{2}
$$


is called joint spectral radius of $\left(a_{1}, \ldots, a_{n}\right)$, where $K$ is one of the joint spectra considered in 1.4 .

2. Joint numerical ranges. We consider joint spatial numerical ranges for operators on a Banach space $X$. For that purpose let

$$
\Pi(X):=\left\{(x, f) \in X \times X^{\prime}: 1=\|x\|=\|f\|=f(x)\right\} .
$$

Given $a_{1}, \ldots, a_{n} \in L(X)$ let

$$
V\left(a_{1}, \ldots, a_{n} ; X\right):=\left\{\left(f\left(a_{1} x\right), \ldots, f\left(a_{n} x\right)\right):(x, f) \in \Pi(X)\right\}
$$

denote the joint spatial numerical range of $a_{1}, \ldots, a_{n}$. Obviously $V\left(a_{1}, \ldots, a_{n} ; X\right)$ is a nonempty and bounded subset of $\mathbb{C}^{n}$.

Our main result (2.2) will state that the convex hull of the joint approximate point spectrum is contained in the closure of the joint numerical range.

A main ingredient in the proof of this result will be the following theorem of Zenger [16] (see [2, p. 20]).

2.1. TheOREM [16]. Let $Y$ be a normed vector space over $\mathbb{C}$, let $y_{1}, \ldots, y_{n}$ be linearly independent vectors in $Y$, and let $\alpha_{k} \geq 0(1 \leq k \leq n)$ such that $\sum_{k=1}^{n} \alpha_{k}=1$. Then there exist $(y, f) \in \Pi(Y)$, and complex numbers $z_{1}, \ldots, z_{n}$ such that $y=\sum_{k=1}^{n} z_{k} y_{k}$ and $f\left(z_{k} y_{k}\right)=$ $\alpha_{k}(1 \leq k \leq n)$.

The following is our main result.

2.2. THeOREM. Let $\left(a_{1}, \ldots, a_{n}\right) \in L(X)^{n}$ denote an $n$-tuple of operators (not necessarily commuting!). Then

$$
\operatorname{conv}\left(A P \sigma\left(a_{1}, \ldots, a_{n} ; X\right)\right) \subseteq V\left(a_{1}, \ldots, a_{n} ; X\right)^{-} .
$$

Proof. Obviously, $A P \sigma\left(a_{1}, \ldots, a_{n} ; X\right) \subseteq V\left(a_{1}, \ldots, a_{n} ; X\right)^{-}$. We next imitate the proof of Crabb's theorem in [2, p. 22]. Let $z^{(j)}=\left(z_{1}^{j}, \ldots, z_{n}^{j}\right) \in A P \sigma\left(a_{1}, \ldots, a_{n} ; X\right)$ $(1 \leq j \leq m)$ and

$$
\delta:=\min \left\{\sum_{k=1}^{n}\left|z_{k}^{j}-z_{k}^{i}\right|: 1 \leq i<j \leq m\right\}, \quad 0<\varepsilon<(4 m n)^{-1} \delta .
$$

By definition of the joint approximate point spectrum we find vectors $x_{1}, \ldots, x_{m} \in X$ such that $\left\|x_{k}\right\|=1$ and

$$
\left\|a_{j} x_{k}-z_{j}^{k} x_{k}\right\|<\varepsilon \quad(1 \leq j \leq n, 1 \leq k \leq m) .
$$

Without loss of generality (by reordering otherwise), we may assume that $\left\{x_{1}, \ldots, x_{m_{0}}\right\}$ is a maximal linearly indepenent subset of $\left\{x_{1}, \ldots, x_{m}\right\}$. Using the Hahn-Banach theorem we find $f_{j} \in X^{\prime}$ such that $1=\left\|f_{j}\right\|$ and $f_{j}\left(x_{i}\right)=\delta_{i j}\left(1 \leq i, j \leq m_{0}\right)$. If $m_{0}<m$, then

$$
x_{m_{0}+1}=\sum_{i=1}^{m_{0}} f_{i}\left(x_{m_{0}+1}\right) x_{i}
$$


and thus

$$
1=\left\|x_{m_{0}+1}\right\| \leq \sum_{j=1}^{m_{0}}\left|f_{j}\left(x_{m_{0}+1}\right)\right| \leq m_{0}
$$

Therefore

$$
\begin{aligned}
\delta(4 m \cdot n)^{-1} & >\left\|a_{1} x_{m_{0}+1}-z_{1}^{m_{0}+1} x_{m_{0}+1}\right\| \\
& =\left\|\sum_{k=1}^{m_{0}} f_{k}\left(x_{m_{0}+1}\right)\left(a_{1} x_{k}-z_{1}^{m_{0}+1} x_{k}\right)\right\| \\
& =\left\|\sum_{k=1}^{m_{0}} f_{k}\left(x_{m_{0}+1}\right)\left(a_{1} x_{k}-z_{1}^{k} x_{k}\right)+\sum_{k=1}^{m_{0}} f_{k}\left(z_{1}^{k}-z_{1}^{m_{0}+1}\right) x_{k}\right\| \\
& \geq\left\|\sum_{k=1}^{m_{0}} f_{k}\left(x_{m_{0}+1}\right)\left(z_{1}^{k}-z_{1}^{m_{0}+1}\right) x_{k}\right\|-m_{0} \delta(4 m \cdot n)^{-1} \quad(\text { by }(+)) .
\end{aligned}
$$

Consequently,

$$
\begin{aligned}
\delta n^{-1}\left|f_{j}\left(x_{m_{0}+1}\right)\right| & \leq\left|f_{j}\left(x_{m_{0}+1}\right)\left(z_{1}^{j}-z_{1}^{m_{0}+1}\right)\right| \\
& =\left|f_{j}\left(\sum_{k=1}^{m_{0}} f_{k}\left(x_{m_{0}+1}\right)\left(z_{1}^{k}-z_{1}^{m_{0}+1}\right) x_{k}\right)\right| \\
& \leq\left\|\sum_{k=1}^{m_{0}} f_{k}\left(x_{m_{0}+1}\right)\left(z_{1}^{k}-z_{1}^{m_{0}+1}\right) x_{k}\right\| \\
& <\delta(4 m n)^{-1}+\delta(4 n)^{-1} \leq \delta(2 n)^{-1},
\end{aligned}
$$

contradicting $(+)$. Therefore $m_{0}=m$.

Next let $\alpha_{k} \geq 0(1 \leq k \leq m), \sum_{k=1}^{m} \alpha_{k}=1$. We apply Zenger's theorem 2.1. This gives us $(x, f) \in \Pi(X)$ with $x=\sum_{k=1}^{m} t_{k} x_{k}$ such that $f\left(t_{k} x_{k}\right)=\alpha_{k} \quad(1 \leq k \leq m)$. Let $z:=$ $\left(z_{1}, \ldots, z_{n}\right)$, where

$$
z_{i}=\sum_{k=1}^{m} \alpha_{k} z_{i}^{(k)}
$$

i.e. $z$ is a convex combination of $z^{(1)}, \ldots, z^{(m)} \in A P \sigma\left(a_{1}, \ldots, a_{n} ; X\right)$. Then

$$
\begin{aligned}
\left|f\left(a_{j} x-z_{j}\right)\right| & =\mid \sum_{k=1}^{m}\left(f\left(t_{k} a_{j} x_{k}\right)-f\left(t_{k} x_{k}\right) z_{j}^{k} \mid\right. \\
& \leq \sum_{k=1}^{m}\left|t_{k}\right|\left\|a_{j} x_{k}-z_{j}^{k} x_{k}\right\|<m . \varepsilon
\end{aligned}
$$

with the same argument as above using $t_{k}=f_{k}(x),\left\|f_{k}\right\|=1(1 \leq k \leq m)$. This proves the theorem. 
Using this and 1.4, we obtain the following result which especially gives a positive answer to the problem of Cho [5] whether $\sigma\left(a_{1}, \ldots, a_{n} ; X\right) \subseteq V\left(a_{1}, \ldots, a_{n} ; X\right)^{-}$is true for an $n$-tuple of pairwise commuting operators.

2.3. Corollary. Let $\left(a_{1}, \ldots, a_{n}\right) \in L(X)^{n}$ denote an $n$-tuple of pairwise commuting operators. Then

$$
\operatorname{conv}\left(\operatorname{Sp}\left(a_{1}, \ldots, a_{n} ;\left\langle a_{1}, \ldots, a_{n}\right\rangle\right)\right) \subseteq V\left(a_{1}, \ldots, a_{n} ; X\right)^{-} .
$$

For $n=1$ the following result is proved in [2, §19 Corollary 5].

2.4. Corollary. Let $(X,\|\cdot\|)$ denote a complex Banach space and let $N(X)$ denote the set of all norms on $X$ equivalent to $\|\cdot\|$. For an $n$-tuple $\left(a_{1}, \ldots, a_{n}\right) \in L(X)^{n}$ of pairwise commuting operators we have

$$
\operatorname{conv}\left(\operatorname{Sp}\left(a_{1}, \ldots, a_{n} ;\left\langle a_{1}, \ldots, a_{n}\right\rangle\right)\right)=\bigcap\left\{V\left(a_{1}, \ldots, a_{n} ;(X, p)\right)^{-}: p \in N(X)\right\} .
$$

Proof. Following Bonsall and Duncan [1, p. 14] let

$$
\begin{gathered}
D\left(L(X), I d_{X}\right):=\left\{f \in L(X)^{\prime}: 1=f\left(I d_{X}\right)=\|f\|\right\}, \\
V\left(L(X) ; a_{1}, \ldots, a_{n}\right):=\left\{\left(f\left(a_{1}\right), \ldots, f\left(a_{n}\right)\right): f \in D\left(L(X), I d_{X}\right)\right\} .
\end{gathered}
$$

Obviously

$$
V\left(a_{1}, \ldots, a_{n} ;(X, p)\right)^{-} \subseteq V\left(L((X, p)) ; a_{1}, \ldots, a_{n}\right) .
$$

Moreover each $p \in N(X)$ induces an operator-norm on $L(X)$ which is equivalent to the original one. On the other hand equivalent norms $q$ on $L(X)$ such that $q\left(I d_{X}\right)=1$ induce equivalent norms on $X$ : Let $f_{0} \in X^{\prime}$ such that $\left\|f_{0}\right\|=1$ and look at the embedding $x \rightarrow f_{0} \otimes x$ from $X$ into $L(X)$. The proof now is an immediate consequence of 2.3 , the above considerations and [1, \$2 Theorem 13].

Concluding Remarks. For $\left(a_{1}, \ldots, a_{n}\right) \in L(X)^{n}$ and $p \in N(X)$ (see 2.4) let

$$
p\left(a_{1}, \ldots, a_{n}\right):=\sup \left\{\left(\sum_{i=1}^{n} p\left(a_{i} x\right)^{2}\right)^{1 / 2}: p(x)=1\right\}
$$

and

$$
v_{p}\left(a_{1}, \ldots, a_{n}\right):=\sup \left\{\left(\sum_{i=1}^{n} f\left(a_{i} x\right)^{2}\right)^{1 / 2}:(x, f) \in \Pi((X, p))\right\}
$$

denote the joint operator norm and the joint numerical radius of $\left(a_{1}, \ldots, a_{n}\right)$ in the Banach space $(X, p)$.

$1^{\circ}$ For a commuting $n$-tuple $\left(a_{1}, \ldots, a_{n}\right) \in L(X)^{n}$ we have by 2.4

$$
r\left(a_{1}, \ldots, a_{n}\right)=\inf \left\{v_{p}\left(a_{1}, \ldots, a_{n}\right): p \in N(X)\right\}
$$

and the infimum is not attained in general. We do not know whether

$$
r\left(a_{1}, \ldots, a_{n}\right)=\inf \left\{p\left(a_{1}, \ldots, a_{n}\right): p \in N(X)\right\}
$$


is true for $n>1$. For $n=1$ equality follows by considering the Neumann series expansion of the resolvent function.

$2^{\circ}$ It follows from 1.4 and 2.3 that

$$
\begin{array}{r}
\operatorname{ext}\left(V\left(a_{1}, \ldots, a_{n} ; X\right)^{-}\right) \\
\subset\left(\mathbb{C}^{n} \backslash S p\left(a_{1}, \ldots, a_{n} ;\left\langle a_{1}, \ldots, a_{n}\right\rangle\right)\right) \cup \operatorname{ext}\left(A P \sigma\left(a_{1}, \ldots, a_{n} ; X\right)\right) .
\end{array}
$$

Consequently $V\left(a_{1}, \ldots, a_{n} ; X\right)^{-}$is convex, if $\left(a_{1}, \ldots, a_{n}\right)$ is jointly convexoid in the sense of Cho and Takagushi [4]. From the results 3.4 and 4.5 in [4] $n$-tuples of doubly commuting hyponormal operators are seen to be jointly convexoid. This is a somewhat weaker result than Dash's [7] which states that $V\left(a_{1}, \ldots, a_{n} ; X\right)$ itself is convex for an $n$-tuple of pairwise commuting normal operators $\left(a_{1}, \ldots, a_{n}\right)$ on a Hilbert space $X$.

$3^{\circ}$ If $(X, p)$ is uniformly convex, $\left({ }^{*}\right)$ can be improved for the "peripheral part" of $V\left(a_{1}, \ldots, a_{n} ; X\right)^{-}$. More precisely, we have

$$
\begin{aligned}
V\left(a_{1}, \ldots, a_{n} ; X\right)^{-} & \cap\left\{z \in \mathbb{C}^{n} ;|z|=p\left(a_{1}, \ldots, a_{n}\right)\right\} \\
& \subset \operatorname{AP} \sigma\left(a_{1}, \ldots, a_{n} ; X\right) ;
\end{aligned}
$$

(see Lumer [9] $(n=1)$ and Cho [5] $(n \geq 1))$.

ACKNOWLEDGEMENT. I thank the referee for his helpful advice.

\section{REFERENCES}

1. F. F. Bonsall and J. Duncan, Numerical ranges of operators on normed spaces and of elements of normed algebras I (Cambridge University Press, 1971).

2. F. F. Bonsall and J. Duncan, Numerical ranges II (Cambridge University Press, 1973).

3. F. F. Bonsall and J. Duncan, Complete normed algebras (Springer Verlag, 1973).

4. M. Cho and M. Takaguchi, Some classes of commuting $n$-tuples of operators, Studia Math. 80 (1984), 245-259.

5. M. Cho, Joint spectra of operators on Banach spaces, Glasgow Math. J. 28 (1986), 69-72.

6. M. Choi and C. Davis, The spectral mapping theorem for joint approximate point spectrum, Bull. Amer. Math. Soc. 80 (1974), 317-321.

7. A. T. Dash, Joint numerical range, Glasnik Mat. Ser. III 7 (1972), 75-81.

8. R. Harte, The spectral mapping theorem in several variables, Bull. Amer. Math. Soc. 78 (1972), 871-875.

9. G. Lumer, Semi-inner-product spaces, Trans. Amer. Math. Soc. 100 (1961), 29-43.

10. J. L. Taylor, A joint spectrum for several commuting operators, J. Functional Analysis 6 (1970), 172-191.

11. J. L. Taylor, The analytic functional calculs for several commuting operators, Acta Math. 125 (1970), 1-38.

12. J. Wermer, Banach algebras and several complex variables, (Springer Verlag, 1976).

13. V. Wrobel, The boundary of Taylor's joint spectrum for two commuting Banach space operators, Studia Math. 84 (1986) 105-111.

14. V. Wrobel, Joint spectra of meromorphic operators, Preprint (Kiel, 1986).

15. W. Zelasko, An axiomatic approach to joint spectra I, Studia Math. 64 (1979), 249-261. 
16. C. Zenger, On convexity properties of the Bauer field of values of matrices, Numer. Math. 12 (1968), 96-105.

Mathematisches Seminar

DER UNIVERSITÄT KIEL

OlShaUSENSTRASSE 40

D-2300 KIEL 1

FED. REP. GERMANY 